\title{
ECM remodeling in hypertensive heart disease
}

\author{
Bradford C. Berk, ${ }^{1}$ Keigi Fujiwara, ${ }^{1}$ and Stephanie Lehoux ${ }^{2}$
}

\author{
${ }^{1}$ Cardiovascular Research Institute and Department of Medicine, University of Rochester, Rochester, New York, USA. "2NSERM U689, Paris, France.
}

\begin{abstract}
Hypertensive heart disease (HHD) occurs in patients that clinically have both diastolic and systolic heart failure and will soon become the most common cause of heart failure. Two key aspects of heart failure secondary to HHD are the relatively highly prevalent LV hypertrophy and cardiac fibrosis, caused by changes in the local and systemic neurohormonal environment. The fibrotic state is marked by changes in the balance between MMPs and their inhibitors, which alter the composition of the ECM. Importantly, the fibrotic ECM impairs cardiomyocyte function. Recent research suggests that therapies targeting the expression, synthesis, or activation of the enzymes responsible for ECM homeostasis might represent novel opportunities to modify the natural progression of HHD.
\end{abstract}

\section{Background}

There is an epidemic of heart failure in the United States. The three major causes of heart failure are hypertensive heart disease (HHD), ischemic heart disease associated with prior myocardial infarction(s), and idiopathic dilated cardiomyopathy. Because the prevalence of hypertension is increasing globally, heart failure secondary to HHD will soon become the most common cause of heart failure. Heart failure is clinically defined by its signs (e.g., peripheral edema, increased heart size, and a third heart sound) and symptoms (e.g., shortness of breath, fatigue, orthopnea, and paroxysmal nocturnal dyspnea). It has become clear that heart failure can clinically present with predominantly diastolic or systolic dysfunction or both. Patients with heart failure secondary to HHD frequently begin their clinical course with only symptoms of diastolic heart failure (in particular, shortness of breath with exertion) but frequently progress to combined diastolic and systolic heart failure. The major difference between HHD and other causes of heart failure can be represented by the manner in which geometric remodeling of the LV occurs (Figure 1). Patients with HHD usually present with LV hypertrophy (LVH) but have a normal-sized LV chamber and preserved systolic function (ejection fraction greater than 50\%). By contrast, patients with heart failure secondary to ischemia or idiopathic cardiomyopathy usually have an enlarged, dilated LV chamber and more frequently also have RV enlargement $(1,2)$.

Pathologic features of hearts from patients with heart failure include cardiomyocyte hypertrophy and death and tissue fibrosis and scarring. Fibrosis seems to be more widespread in HHD than in other causes of heart failure. It is found throughout the heart, including the anterior, posterior, and lateral walls of the LV; the interventricular septum; and even the RV. Because of fibrosis, the classic finding in HHD is increased myocardial stiffness, especially during diastole. Although fibrosis contributes to stiffness, it is the quality of the ECM, not the quantity, that is most important. Importantly, fibrosis disrupts the coordination of myocardial excitation-contraction coupling in both systole and diastole. In the healthy heart, cardiomyocytes are

Nonstandard abbreviations used: ADAM, a disintegrin and metalloproteinase; BMP1, bone morphogenetic protein 1; ET-1, endothelin 1; HB-EGF, heparin-binding EGF; HHD, hypertensive heart disease; LVH, LV hypertrophy; PAK1, p21-activated kinase 1; RAAS, renin-angiotensin-aldosterone system; SHR, spontaneously

hypertensive rat; TIMP, tissue inhibitor of metalloproteinases.

Conflict of interest: The authors have declared that no conflict of interest exists. Citation for this article: J. Clin. Invest. 117:568-575 (2007). doi:10.1172/JCI31044. connected together in an electrical synctium that permits a temporally coupled contraction. Transmission of the systolic contraction is facilitated by a scaffold of type I and type III fibrillar collagens, which are the major components of the cardiac ECM. Following contraction, there is an active relaxation process during diastole. Weber and Shirwany (3) have noted that the tensile strength of type I collagen is similar to that of steel, making it obvious that the ECM is the major determinant of myocardial stiffness during diastole.

Alterations in HHD that contribute to disease pathology other than those in the ECM include changes in the number and function of other resident cells, myocardial apoptosis, and changes in calcium handling associated with impaired relaxation. Importantly, there are also substantial changes in the peripheral vasculature (especially resistance vessels) that impair cardiac function. Little and colleagues (4) showed that patients who present with pulmonary edema with preserved systolic function have HHD characterized by severe peripheral vascular stiffness. The impaired properties of the aorta and resistance arterioles (the dominant determinants of vascular tone and pressure) contribute importantly to cardiac dysfunction in HHD. However, this Review focuses on the functional and structural changes in ECM in the heart that characterize HHD. The features of HHD ECM are discussed in the context of alterations in the cellular and hormonal environments that lead to changes in ECM turnover and a profibrotic state. Major features of the model we propose for the development of HHD include the early transition of cardiac fibroblasts to myofibroblasts (Figure 2). Myofibroblasts produce a different ECM than fibroblasts and modify the balance of MMPs and their inhibitors (tissue inhibitors of metalloproteinases [TIMPs]) to promote fibrosis. The change in ECM modifies the signals that cardiac myocytes receive from their scaffolding environment, leading to changes in gene expression associated with hypertrophy and contractile dysfunction. Finally, activation of the renin-angiotensin-aldosterone system (RAAS) and increased levels of active TGF- $\beta 1$ recruit smooth muscle cells, monocytes, and fibroblasts and stimulate a genetic program of wound repair and ECM deposition, leading to perivascular fibrosis and amplification of the profibrotic state.

\section{What drives HHD?}

In the Framingham Heart Study, echocardiographic LVH was found to be present in $15 \%$ of the population and was independently associated with several cardiovascular endpoints, including 

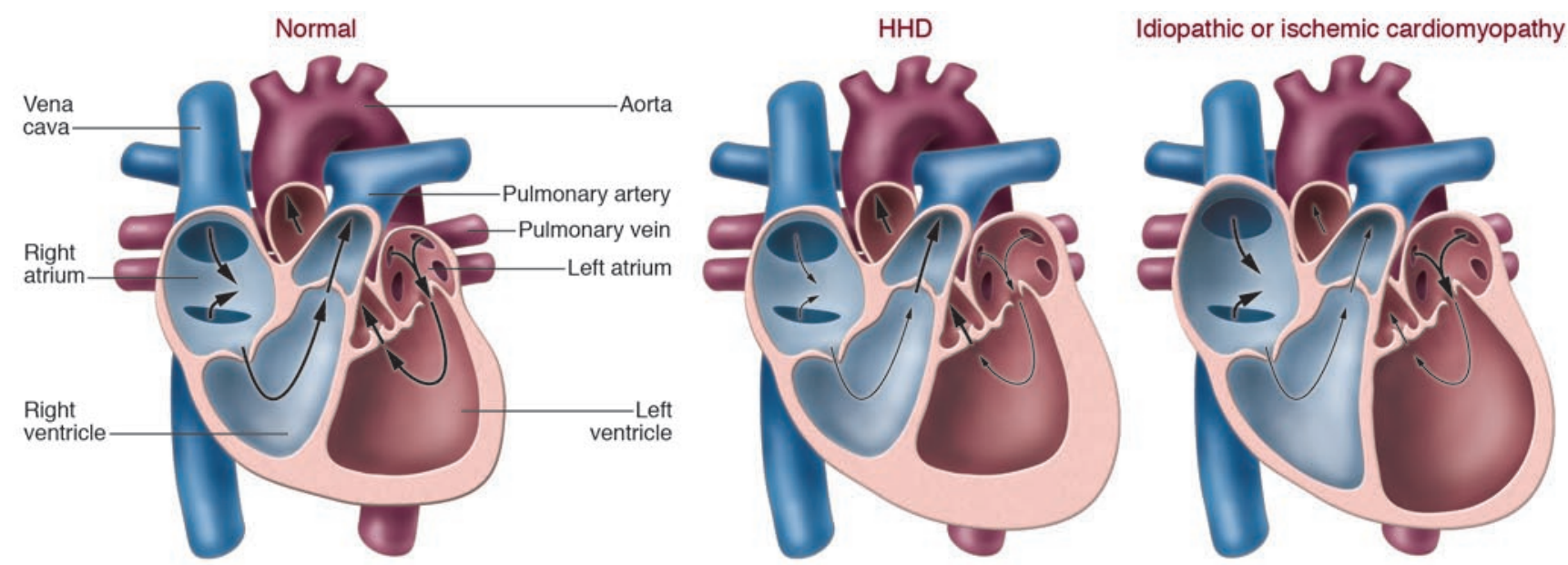

Figure 1

Schematic representation of changes in the cardiac chambers of an individual with HHD compared with idiopathic or ischemic cardiomyopathy. The main difference between HHD and the other two main causes of heart failure (ischemic heart disease associated with prior myocardial infarction[s] and idiopathic dilated cardiomyopathy) is the nature of the geometric remodeling of the LV chamber. Patients with HHD usually present with LVH but with a normal-sized LV chamber and preserved systolic function. By contrast, patients with heart failure secondary to ischemia or idiopathic cardiomyopathy usually have an enlarged, dilated LV chamber and more frequently also have RV enlargement.

coronary heart disease and stroke (5). Importantly, after adjusting for other cardiovascular disease risk factors, including blood pressure, $\mathrm{LVH}$ is associated with a doubling in mortality in both white and African American cohorts (5). As would be expected, there are substantially more cardiovascular events in hypertensive patients who have $\operatorname{LVH}(5,6)$. It is reasonable to propose that the development of LVH associated with HHD might represent a protective mechanism, providing compensatory power to allow the heart to withstand the hemodynamic strain associated with increased arterial pressure. However, the continued presence of LVH leads to cardiac dysfunction manifest by a reduction of coronary flow reserve, tissue ischemia, development of arrhythmias, heart failure, and sudden death (7). There is a strong correlation between blood pressure and LVH; specifically, high blood pressure is associated with a 10 -fold increase in the incidence of LVH detected by electrocardiography (8). Blood pressure-independent effects also contribute to $\mathrm{LVH}$, since antihypertensive therapies differ in their ability to reduce $\mathrm{LVH}$, despite similar efficacy in blood pressure reduction $(5,9)$. Furthermore, RV hypertrophy coexists with LVH despite a lack of hemodynamic strain (10). Nonhemodynamic factors that are likely to influence HHD include hormones and cytokines (such as the RAAS, TGF- $\beta 1$, TNF- $\alpha$, and IL- 1 ) that lead to a profibrotic and inflammatory environment (9).

\section{Structural changes in HHD}

A fundamental characteristic of hypertensive cardiac remodeling is myocardial stiffness, which is associated with fibrosis, altered contractile and relaxation properties, and changes in cardiac cellularity (especially perivascular inflammation). The scaffolding of cardiomyocytes is provided by a network of fibrillar collagen (Figure 3) (11). Based on morphology, the network can be subdivided into three components. The epimysium is located on the endocardial and epicardial surfaces of the myocardium, where it provides support for endothelial and mesothelial cells. The perimysium surrounds muscle fibers, and perimysial strands connect groups of muscle fibers together. The endomysium arises from the perimysium and surrounds individual muscle fibers. Struts of endomysium tether muscle fibers together and function as the sites for connections to cardiomyocyte cytoskeletal proteins across the plasma membrane (e.g., laminin to dystroglycan; see below). The endomysium is also the source of ECM scaffolding for blood vessels. Morphologically, fibrotic tissue in the heart is visualized as perivascular fibrosis involving the intramural coronary arterial vasculature, interstitial fibrosis (accumulated perimysium), and microscopic scarring (Figure 3) (12). The process of fibrosis has several different stages, which is pertinent to therapeutic options since it is probable that fibrosis is reversible (at least, prior to scarring). It is also reasonable to propose that optimal treatment strategies will differ according to the level of disease progression.

Changes in the collagen network present in HHD impair both systolic and diastolic function (13). Collagen is a stable protein whose balanced turnover (synthesis and degradation) by cardiac fibroblasts is normally slow (estimated to be $80-120$ days) (3). Collagen turnover is primarily regulated by fibroblasts during normal physiology. However, under pathologic conditions, morphologically distinct cells termed myofibroblasts appear (Figures 2 and 3). These cells are defined by their dual functions: fibroblast-like in terms of ECM synthesis and smooth muscle myocyte-like in terms of migration. Myofibroblast-mediated collagen turnover is regulated by autocrine and paracrine factors generated within the myocardium and by endocrine hormones derived from the circulation.

In animal models of HHD, an increase in interstitial collagen (accumulated perimysium) is associated with diastolic heart failure, whereas degradation of endomysial and perimysial components of the collagen scaffolding is accompanied by ventricular dilatation and systolic heart failure (14). These data suggest that the transition from compensated LVH to heart failure is associated with degradation of ECM. Loss of the collagen network might cause systolic dysfunction by at least three mechanisms (13). The first mechanism involves interruptions in the collagen matrix that provides support, geometric alignment, and coordination of contraction by cardiomyocyte bundles. The second mechanism 


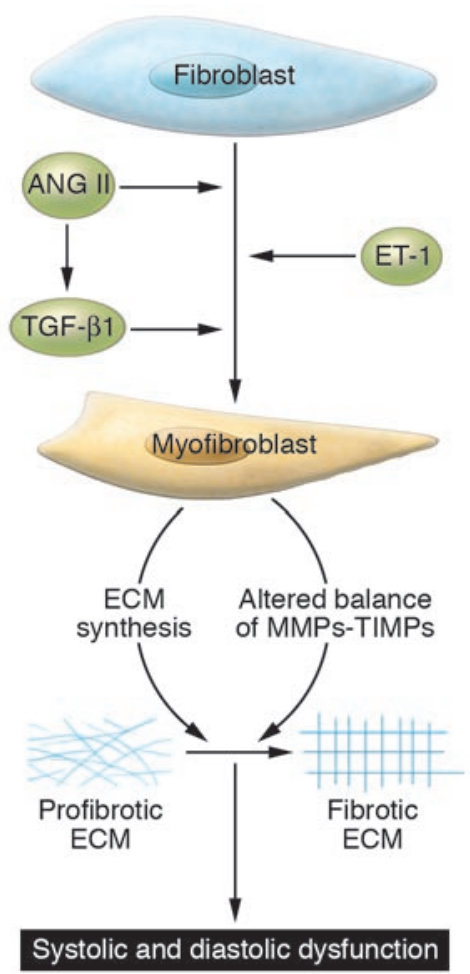

Figure 2

Mechanisms for transition of fibroblasts to myofibroblasts. The transition of fibroblasts to myofibroblasts is an early event in HHD, regulated in part by increased expression of the hormones of the RAAS system (renin, ANG II, and aldosterone), ET-1, and TGF- $\beta 1$. Myofibroblasts express a gene program that contributes to a progressive profibrotic state. Changes in the ECM occur in part due to an altered balance of MMPs and their inhibitors (TIMPs). These changes lead to a stiffening of the ECM and functional alterations that cause changes in signaling to myocytes. The altered physical and functional environment of the myocytes leads to progressive cardiac dysfunction.

involves the loss of the normal interactions between endomysial components such as laminin and collagen with their receptors (dystroglycans and integrins), which is required for contractile synchrony and long-term cardiomyocyte homeostasis. The third mechanism is the sliding displacement (slippage) of cardiomyocytes, leading to a decrease in the number of muscular layers in the ventricular wall and to $\mathrm{LV}$ dilation.

$E C M$ synthesis in HHD. In fibrotic conditions there is evidence that both the synthesis and degradation of collagens are altered, yielding an accumulation of collagen that (together with enhanced crosslinking) results in fibrosis. Myocardial stiffness is mostly attributable to changes in the composition and arrangement of ECM proteins, including type I and type III fibrillar collagens $(15,16)$. Procollagen is synthesized by fibroblasts (and myofibroblasts in disease) and secreted into the pericellular space, where it forms collagen fibrils that assemble into fibers. Myofibroblast collagen turnover is regulated by a number of growth factors such as ANG II, TGF- $\beta 1$, IGF-1, and TNF- $\alpha$ (17), some of which act in concert. For example, ANG II produced locally by activated macrophages and myofibroblasts regulates their expression of TGF- $\beta 1$, a fibrogenic cytokine that, in turn, upregulates the expression of the genes encoding type I and type III fibrillar collagen $(18,19)$. Besides collagen, expression of many other ECM components, including elastin, fibrillin, fibronectin, and proteoglycans, are also changed in HHD.

In humans, a reduction in circulating markers of collagen degradation occurs early after the onset of antihypertensive treatment and parallels the reduction in hemodynamic load (20), similar to what has been found in animals (21). Spontaneously hypertensive rats (SHRs) show greater pro-collagen type I levels in the myocardium than normotensive Wistar-Kyoto rats, indicative of increased collagen type I synthesis in the hypertensive animals $(22,23)$. This is also accompanied by increased collagen crosslinking following the development of cardiac hypertrophy in SHRs (24). It should be noted that increases in blood pressure per se have powerful effects on the synthesis of protein by both cardiomyocytes and fibroblasts. When pressure is rapidly increased in rodent hearts, collagen and total protein synthesis increase and protein degradation decreases within 3 hours $(25,26)$. In most studies there is a gradual return to baseline synthesis in two to three weeks, associated with protein degradation and decreased fibrosis (27). These data suggest that even intermittent hypertension might lead to changes in ECM and fibrosis.

ECM degradation in HHD. During the initial phases of HHD described above, the predominant process is increased synthesis of cardiomyocyte and ECM proteins. Much less is known about the subsequent phases of HHD, especially the transition from the compensated state to clinically apparent heart failure. The natural response of the body to increased synthesis of ECM components is to increase the levels and activity of enzymes that degrade the ECM. However, degradation of ECM in a heart that has undergone hypertrophy might not be benign. Recently, Diez and colleagues provided information regarding collagen degradation in patients with HHD during the process of deteriorating systolic function (28). They showed that enhanced MMP-mediated collagen degradation contributed to the LV dilation and decline in ejection fraction seen with systolic heart failure in HHD. Specifically, they found in patients with systolic heart failure that perivascular fibrosis and scarring occupied a greater portion of the myocardium, while the number of interstitial collagen fibers was reduced. Based on these findings they proposed that an imbalance in the ratio of MMPs to their inhibitors, the TIMPs, might underlie LV dilation and reduced ejection fraction in systolic heart failure. These data stress the importance of studying the mechanisms responsible for ECM degradation as well as those responsible for ECM synthesis.

The heart contains many MMPs that can degrade ECM proteins with differing degrees of specificity. These include the collagenases (MMP1, MMP8, and MMP13) that initiate the ECM degradation process by cleaving the $\alpha$-chains of type I and type II collagens and the gelatinases (MMP2 and MMP9) that further process collagen fragments $(29,30)$. Although some MMPs are constitutively expressed, the expression of others is regulated by hormones, growth factors, cytokines, and mechanical strain $(31,32)$. MMPs are generally synthesized as inactive precursors that are then activated by serine proteases, secreted MMPs, or the highly related membrane-type MMPs (33). Localization of MMPs, either membrane attached or secreted, can determine their relative activity (30). MMP activation can contribute to the fibrotic process by participating in a vicious circle in which ECM degradation promotes ECM protein synthesis and fibrosis. This pathway is particularly detrimental because the nature and organization of the newly synthesized ECM differs from that of the native ECM. 


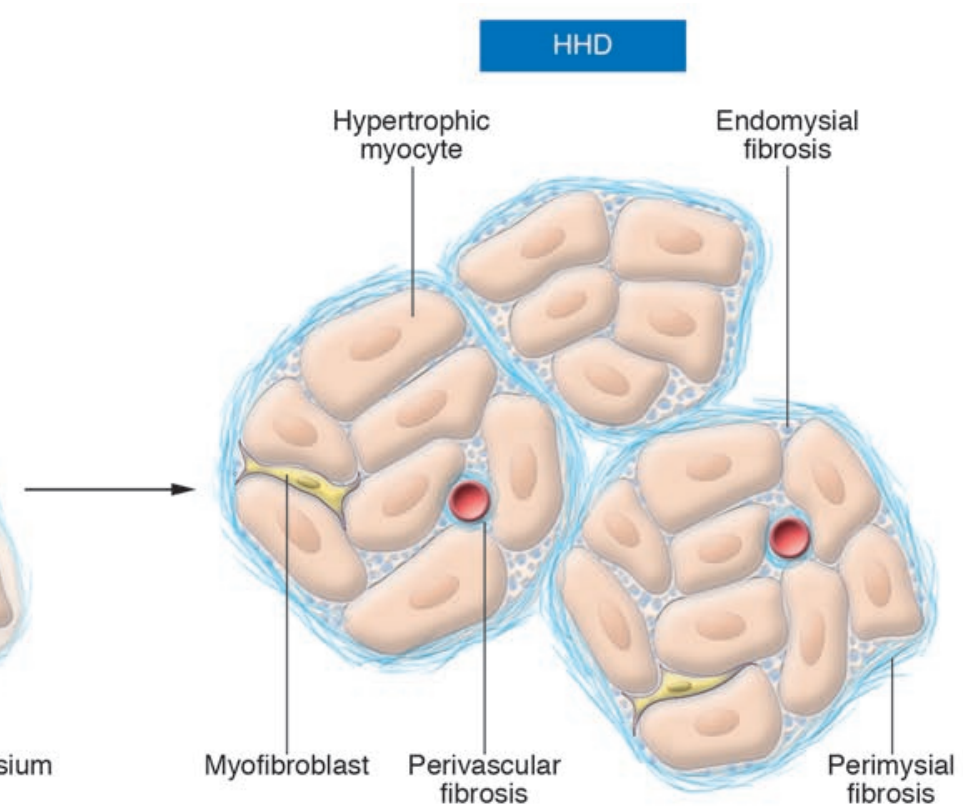

\section{Figure 3}

Schematic representation of changes to the collagen network in HHD. In the normal heart, thin layers of perimysium and endomysium surround myocardial bundles and myocytes, respectively. The walls of the blood vessels also contain adventitial fibroblasts that create an endomysial network. In HHD, there is hypertrophy of cardiomyocytes and transition of fibroblasts to myofibroblasts. These changes are associated in early disease with increases in ECM manifest by perivascular fibrosis and fibrosis of the endomysium and perimysium.

For example, turnover of oxidized and highly crosslinked collagen (which is more prevalent during fibrosis) is slower than that of normal collagen, favoring the accumulation of the former, a much stiffer collagen (34). Other potential mediators of ECM degradation include the membrane-bound proteases known as a disintegrin and metalloproteinases (ADAMs) and their soluble thrombospondin motif-containing counterparts (ADAMTSs), both of which can participate in the release of growth factors from the ECM. Importantly, these enzymes have been shown to increase levels of proteins such as heparin-binding EGF (HB-EGF) and TGF- $\alpha$. TGF- $\alpha$, which is cleaved and activated by ADAM17, has been shown to influence ECM homeostasis in the pressureoverloaded heart $(35,36)$.

Studies regarding the roles of specific MMPs and TIMPs in HHD are confusing due to the simplicity of animal models compared with patients with HHD. In hypertensive Dahl salt-sensitive rats, MMP2, TIMP1, and TIMP2 expression levels increase as LVH progresses (14). Similarly, MMP2 activity increases in SHRs (37), and cyclic stretching of cardiomyocytes elevates mRNA expression, protein synthesis, and the activity of MMP2 and MMP14 (38). A role for MMPs in hypertensive cardiac remodeling has also been strongly demonstrated using MMP2-deficient mice. In a model of aortic banding, these animals demonstrated lower LV weight and LV end-diastolic pressure and showed less interstitial fibrosis and cardiomyocyte hypertrophy than wild-type controls with similar levels of aortic hypertension (39). In other studies, mice lacking either MMP9 or urokinase-like plasminogen activator were also protected from cardiac fibrosis and dysfunction following pressure overload (40). Further evidence for the importance of MMP9 is provided by the observations that rising plasma MMP9 levels correlated with deterioration of LV function (41) and that enhanced MMP9 activity (but not MMP2 activity,
TIMP1-TIMP4 expression, or collagen crosslinking) corresponded with the transition from compensated LVH to congestive heart failure (42). Pharmacologic data also demonstrate a deleterious role for MMP activity in HHD, since treatment with a broad-spectrum MMP inhibitor completely prevented the transition to overt heart failure in rats with spontaneous hypertensive heart failure (43). Similar observations were made in the Dahl salt-sensitive rat model of pressure overload and heart failure (44).

In hypertensive patients with LVH, there are increased levels of circulating TIMP1 but diminished levels of circulating MMP1 and collagen type I telopeptide (CITP, a breakdown product of collagen) (45), compared with hypertensive patients without LVH. More recently, Ahmed et al. showed that patients with hypertension but normal LV structure and function had normal plasma MMP and TIMP levels (29). By contrast, patients with hypertension and LVH had decreased levels of MMP2 and MMP13 and increased levels of MMP9. Only patients with LVH and heart failure had increased levels of TIMP1. Based on these data, they concluded that decreased ECM degradation was associated with LVH and diastolic dysfunction. These studies highlight a potential beneficial effect of specific MMP9 inhibitors in HHD and raise the general concept that altering the balance of degradation and synthesis of ECM might have clinical utility.

ECM regulation of myocyte and myofibroblast function. A disordered balance of MMP and TIMP activity in HHD would exert profound effects on cardiac function in several ways. First, cell-cell junctions are subject to MMP-mediated degradation. Depending on the type of MMP expressed and the composition of the cell-cell junctions, different junctions would be more susceptible to degradation. For example, direct binding and processing of connexin- 43 by MMP7 has been related to arrhythmias and myocardial dysfunction in mice (46). Second, given the dynamic nature of intercellular adhe- 


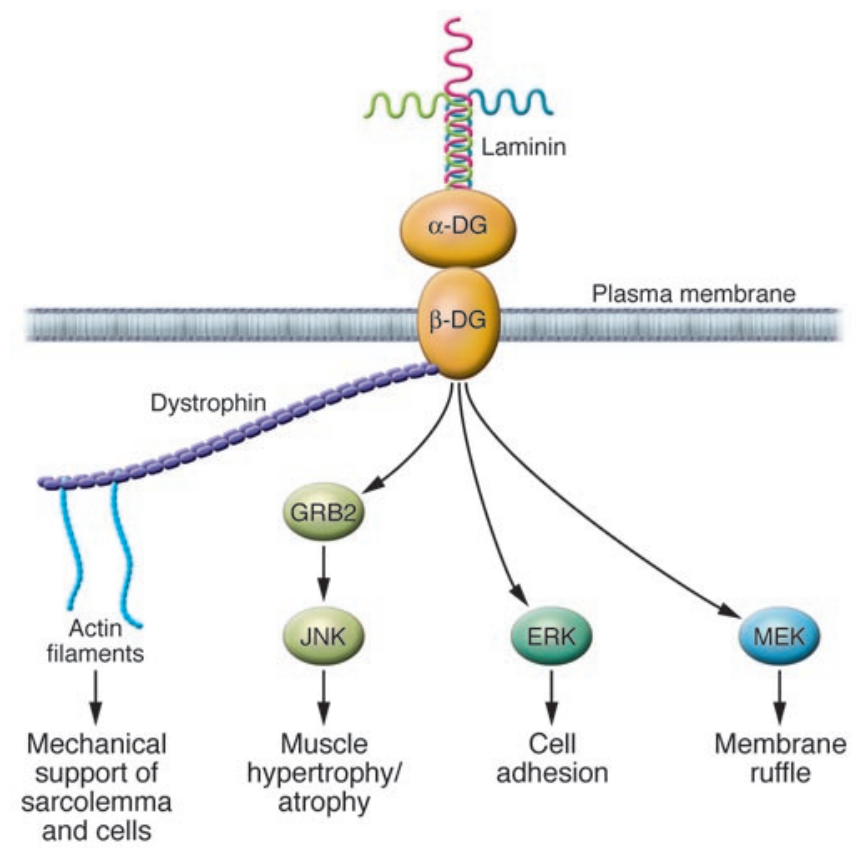

Figure 4

A laminin-dystroglycan-dystrophin signaling cascade. Dystroglycan (DG) is a key component of the dystrophin-associated glycoprotein complex that provides mechanical support to the sarcolemma. $\beta$-Dystroglycan is the transmembrane subunit, and its extracellular domain noncovalently binds $\alpha$-dystroglycan. Laminin is a major component of the basal laminae and a prominent protein in the endomysium. The binding of laminin to dystroglycan activates a growth factor receptor-bound protein 2-RAC1-PAK1-JNK (GRB2-RAC1-PAK1-JNK) pathway that promotes hypertrophy, an initial adaptive response to increased pressure. Decreased expression of laminin, as might occur during the transition to heart failure, might impair survival signaling to cardiomyocytes and predispose them to apoptosis, similar to the pathology in skeletal muscle dystrophies. Direct association between dystroglycan and MEK and between dystroglycan and ERK was recently demonstrated (82). MEK-dystroglycan association was localized to membrane ruffles, while ERK-dystroglycan association was found in focal adhesions. It is not known how these interactions are regulated.

sions in cell signaling and cytoskeletal biology (e.g., activation of PI3K, mechanotransduction, RAC activation, and microtubule polymerization), it is critical to elucidate the effects of decreased intercellular connections. Third, peptides derived from ECM macromolecule degradation, termed matrikines, modulate proliferation, migration, and MMP expression by cells present in the heart, including smooth muscle cells and myofibroblasts $(47,48)$. The best characterized matrikines are derived from elastin by enzymes such as MMPs (MMP2, MMP7, MMP9, and MMP12), elastases, and cathepsins $(47,49,50)$. Fourth, MMPs act on non-ECM proteins, cleaving (and potentially activating) a number of remodeling modulators such as EGF, HB-EGF, and TNF- $\alpha$ (51).

Changes in ECM components and MMP activity also influence cardiac contractile function by influencing bidirectional signaling from integrins (i.e., inside out signaling as well as outside in signaling) and the coordination of integrin signaling with other receptors (e.g., modulation of growth factor signaling) $(52,53)$. One such example relates to the interaction between the integrin $\alpha_{v} \beta_{1}$ and TGF- $\beta 1$. Overexpression of $\alpha_{v} \beta_{1}$ stimulates TGF- $\beta 1$ expression, whereas TGF- $\beta 1$ can increase expression of $\alpha_{v} \beta_{1}(51)$. Other examples include changes in cell survival pathways. For example, deletion of melusin, a mechanosensitive integrin $\beta_{1^{-}}$ interacting protein, accelerates hypertensive LVH and heart failure, whereas overexpression of the protein has the opposite effect, through activation of antiapoptotic AKT and ERK1/2 signaling pathways (54). Mechanical forces related to hypertension-induced myocardial stretch constitute a key activator of intracellular signaling pathways such as those involving phospholipase C, phospholipase D, and phospholipase A2; PKC; tyrosine kinases; p21 MAPKs; and 90-kDa S6 kinase, potentially through the release of growth-promoting factors (ANG II, endothelin 1 [ET-1], and phenylephrine) or by integrin activation (51). Apart from promoting fibroblast proliferation, a number of these factors can also increase expression of MMPs (51). For example, in vascular smooth muscle cells, PDGF-BB and IGF-1 increase the transcription rate of $M m p 2$ through activation of PI3K and its downstream effector, AKT (55).

To provide an example of how alterations in ECM proteins can influence cardiomyocyte function, we discuss the nature of signaling from laminin to dystroglycan-dystrophin (Figure 4). Although the dystroglycan-dystrophin pathway has been extensively studied in muscular dystrophies, its role in HHD has been little studied $(56,57)$. Dystroglycan is best known for its interaction with dystrophin in striated muscle and is a key component of the dystrophin-associated glycoprotein complex that provides mechanical support to the sarcolemma. $\beta$-Dystroglycan is the transmembrane subunit, and its extracellular domain noncovalently binds $\alpha$-dystroglycan, a heavily glycosylated extracellular peripheral membrane protein (58). Laminin is a major component of the basal laminae throughout the body and a prominent protein in the endomysium (59). In the heart, dystrophin signaling seems to be important for the response to pressure, since pressure overload increases dystrophin expression and since dystrophindeficient mice exhibit increased myocardial apoptosis and fibrosis in response to acute pressure overload $(56,57)$. Since laminin binding to dystroglycan activates a growth factor receptor-bound protein 2-RAC1-p21-activated kinase 1-JNK (GRB2-RAC1-PAK1JNK) pathway that promotes hypertrophy, it is probable that this pathway is required for the response to increased pressure (60). This exemplifies the importance of signaling from the ECM to cardiomyocytes for physiologic compensation in response to stresses such as hypertension.

\section{Signaling cascades influencing HHD}

Fibroblast transition to myofibroblasts. The mechanisms responsible for the transition of fibroblasts to myofibroblasts have been recently elucidated (Figure 2). Myofibroblasts are smooth muscle-like fibroblasts that express $\alpha$-SMA and contain a contractile apparatus composed of actin filaments and associated proteins organized into prominent stress fibers. Myofibroblast formation is controlled by growth factors, cytokines, and mechanical stimuli (61). Key hormones and cytokines for this transition are ANG II, ET-1, and TGF- $\beta 1$ (Figure 2). TGF- $\beta 1$ is a critical factor because it stimulates both myofibroblast formation and collagen production. A recent study showed that increasing cAMP blocked the fibroblast-to-myofibroblast transition, perhaps due to a RhoAdependent effect $(18,62)$. ANG II is also very important because it sensitizes fibroblasts to this transition by directly promoting TGF- $\beta 1$ signaling, elevating SMAD3 levels, and inducing nucle- 


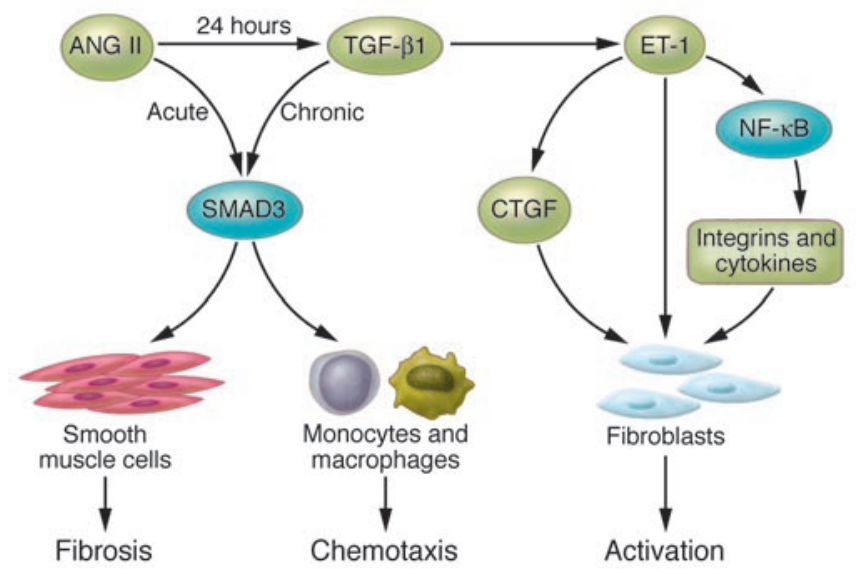

Figure 5

Common signaling pathways activated by ANG II, ET-1, and TGF- $\beta 1$ contribute to fibrosis. SMAD3 mediates acute and chronic changes in gene expression that lead to inflammation and fibrosis. Chronic exposure to ANG II increases expression of TGF- $\beta 1$. This cytokine promotes a profibrotic environment by multiple mechanisms, including stimulation of SMAD3-dependent gene expression in smooth muscle cells and monocytes and increased expression of ET-1 by endothelial cells. ET-1 promotes fibroblast activation by stimulating connective tissue growth factor (CTGF) expression and activating an NF-kB-dependent gene program that is profibrotic.

ar translocation of phosphorylated SMAD3 $(63,64)$ (Figure 5). As discussed below, this might be particularly important for the chronic effects of TGF- $\beta 1$ on ECM production.

Effects of the RAAS. There is evidence that all major components of the RAAS - renin, ANG II, and aldosterone - exert profibrotic effects on cells. Although there are limited data regarding renin, a recent study by Huang et al. (65) showed that both renin and prorenin increased the synthesis of TGF- $\beta 1$ in mesangial cells. Renin also enhanced the synthesis of fibronectin, collagen I, and plasminogen activator inhibitor-1. The actions of renin seemed to be independent of ANG II, based on the inability of the angiotensin-converting enzyme inhibitor enalaprilat and the ANG II type 1 receptor blocker losartan to block TGF- $\beta 1$ synthesis. Data regarding expression of renin and prorenin receptors in cardiac fibroblasts are not yet available, so the role of renin in cardiac fibrosis and HHD remains unclear.

The greatest weight of evidence indicates that ANG II is the dominant hormone responsible for cardiac fibrosis in HHD. ANG II exerts its effects directly through the ANG II type 1 receptor and indirectly through induction of TGF- $\beta 1$ (17). SMAD 3 seems to be a common pathway for these two mechanisms, as summarized by Sorescu (64) and Wang (63) (Figure 5). According to a recent review of ANG II signaling by Mehta and Griendling (66), there are three rapid pathways activated by ANG II that lead to the expression of genes encoding ECM components, with JNK and activator protein 1 (AP-1) activation as a final common pathway. The first is an ROS pathway dependent on the small G-proteins Rho and RAC; the second is a focal adhesion kinase (FAK) pathway dependent on calcium, c-SRC, and paxillin; and the third is a PAK pathway. Studies by Shen et al. (41) that involved our laboratory showed that ANG II induced adventitial fibroblast differentiation to myofibroblasts by a pathway that involved NADPH oxidase generation of ROS and activation of p38 MAPK and JNK pathways. A second pathway is the tyrosine kinase pathway mediated by c-SRC. After activation, c-SRC translocates to focal adhesions and initiates phosphorylation of FAK1 and proline-rich tyrosine kinase 2 (PYK2). Subsequently, the small GTPase RAC is activated at the focal adhesion and promotes JNK activation. The final pathway involves PAK, which has been shown to stimulate RAC and thereby activate JNK $(67,68)$.

In addition to these rapid pathways, there are several indirect pathways for ANG II-mediated signal transduction that probably regulate ECM turnover. Two important pathways result in the activation of MMPs and the secretion and activation of TGF- $\beta 1$. ANG II has been shown to transactivate tyrosine kinase receptors such as the EGF receptor (69). The predominant mechanism involved in this process seems to be stimulation of ADAM17, which cleaves matrix and cell-bound HB-EGF to generate soluble HB-EGF locally (70). HB-EGF secreted by cardiomyocytes leads to cellular growth and reduced expression of the principal ventricular gap junction protein, connexin 43 . The local disruption in gap junctions might be a part of the hypertrophic response induced by HB-EGF (71) and might have functional consequences to impair electrical coupling of cardiomyocytes. However, for myofibroblasts, the dominant pathway seems to be through the secretion and activation of TGF- $\beta 1$. TGF- $\beta 1$ is secreted as a large latent complex bound to its cleaved prodomain and, through this domain, to latent TGF- $\beta 1$ binding proteins (72). The latter target large latent complexes of TGF- $\beta 1$ to the ECM (72). Bone morphogenetic protein 1-like (BMP1-like) metalloproteinases have key roles in ECM formation, where they convert TGF- $\beta 1$ precursors into mature functional proteins (73). Recently, Ge and Greenspan (73) showed that BMP1 cleaves latent TGF- $\beta 1$-binding protein 1 at two specific sites, thereby liberating large latent complexes of TGF- $\beta 1$ from the ECM and activating TGF- $\beta 1$ through cleavage of the latency-associated peptide by non-BMP1-like proteinases.

In addition to affecting TGF- $\beta 1$ secretion and activation, ANG II also directly enhances TGF- $\beta 1$ signaling by increasing SMAD2 levels and augmenting nuclear translocation of phosphorylated SMAD3 $(63,64)$ (Figure 5). Specifically, Wang et al. showed that 24 hours after ANG II stimulation, the SMAD2/3 signaling pathway was activated; this pathway was TGF- $\beta 1$ dependent because it was blocked by a TGF- $\beta 1$-specific antibody and by overexpression of a dominant-negative TGF- $\beta 1$ receptor (63). Wang et al. also found that activation of SMAD3 but not SMAD2 was critical because ANG II induced SMAD3/4 promoter activities and collagen-matrix expression was abolished in vascular smooth muscle cells lacking SMAD3 but not SMAD2.

A role for aldosterone in cardiac fibrosis has become increasingly apparent, especially in light of pharmacologic aldosterone inhibitor studies with spironolactone and eplerenone (74-76). A recent study in SHRs by Susic et al. (77) indicated that eplerenone can directly reduce fibrosis, independently of its hemodynamic effects. Specifically, eplerenone reduced collagen in the RV, a chamber not exposed to systemic hemodynamic overload. The exact mechanism by which antagonizing the mineralocorticoid receptor (that binds aldosterone), prevents fibrosis is unknown. Possible mechanisms include alterations in intracellular signaling, changes in activation of transcription and growth factors, decreased ET-1 production, increased endothelial NO production, and decreased oxidative stress.

Direct effects of TGF- $\beta 1$. Expression of mRNA encoding TGF- $\beta 1$ is increased in the LV myocardium of patients with LVH and dilated cardiomyopathy (78). TGF- $\beta 1$ is specifically expressed 
in hypertrophic myocardium during the transition from stable hypertrophy to heart failure (10). In vitro, TGF- $\beta 1$ induces the production of ECM components including fibrillar collagen, fibronectin, and proteoglycans by cardiac fibroblasts (79) and stimulates fibroblast proliferation and phenotypic conversion to myofibroblasts (61). In addition, TGF- $\beta 1$ self amplifies its expression in myofibroblasts (80). Overexpression of TGF- $\beta 1$ in transgenic mice results in cardiac hypertrophy that is characterized by both interstitial fibrosis and hypertrophic growth of cardiac myocytes (81).

\section{Summary}

In patients with HHD, changes in the ECM that predispose to fibrosis contribute importantly to the functional and structural abnormalities that cause progressive cardiac dysfunction. In patients with hypertension, normal MMP and TIMP profiles correlate with normal LV structure and function. Changes in MMP profiles that favor ECM accumulation are associated with LVH and diastolic dysfunction (29), and increases in ECM degradation seem to herald the transition to systolic failure. These findings suggest that monitoring plasma markers of myocardial ECM remodeling might provide important prognostic information with respect to ongoing adverse LV remodeling in patients with HHD. In conclusion, ECM-generating and -degrading enzymes have an established role in both the development and the progression of HHD. Recent research suggests that therapies that target expression, synthesis, or activation of these enzymes might represent novel opportunities to alter the natural history of $\operatorname{HHD}(35,36)$. The first molecules tested as ECM-modifying drugs have been the MMP inhibitors. Unfortunately, these molecules have been limited in their development by side effects such as tendonitis. Furthermore, because of the widespread expression of ECM components in all tissues, development of ECM drugs specific for individual organs will clearly be challenging.

\section{Acknowledgments}

This work was supported by NIH grants HL49192 and HL63462 (to B.C. Berk) and European Vascular Genomics Network grant FP6-LSHM-CT 2003-S03254 (to S. Lehoux).

Address correspondence to: Bradford C. Berk, Cardiovascular Research Institute, Box 679, 601 Elmwood Ave., University of Rochester, Rochester, New York 14642, USA. Phone: (585) 273-1946; Fax: (585) 273-1497; E-mail: bradford_berk@urmc.rochester.edu.
1. Zile, M.R., and Brutsaert, D.L. 2002. New concepts in diastolic dysfunction and diastolic heart failure: part II: causal mechanisms and treatment. Circulation. 105:1503-1508.

2. Zile, M.R., and Brutsaert, D.L. 2002. New concepts in diastolic dysfunction and diastolic heart failure: part I: diagnosis, prognosis, and measurements of diastolic function. Circulation. 105:1387-1393.

3. Shirwany, A., and Weber, K.T. 2006. Extracellular matrix remodeling in hypertensive heart disease. J. Am. Coll. Cardiol. 48:97-98.

4. Gandhi, S.K., et al. 2001. The pathogenesis of acute pulmonary edema associated with hypertension. N. Engl. J. Med. 344:17-22.

5. Benjamin, E.J., and Levy, D. 1999. Why is left ventricular hypertrophy so predictive of morbidity and mortality? Am. J. Med. Sci. 317:168-175.

6. Liao, Y., Cooper, R.S., Durazo-Arvizu, R., Mensah, G.A., and Ghali, J.K. 1997. Prediction of mortality risk by different methods of indexation for left ventricular mass. J. Am. Coll. Cardiol. 29:641-647.

7. Diez, J., Lopez, B., Gonzalez, A., and Querejeta, R. 2001. Clinical aspects of hypertensive myocardial fibrosis. Curr. Opin. Cardiol. 16:328-335.

8. Levy, D. 1988. Left ventricular hypertrophy. Epidemiological insights from the Framingham Heart Study [review]. Drugs. 35(Suppl. 5):1-5.

9. Kenchaiah, S., and Pfeffer, M.A. 2004. Cardiac remodeling in systemic hypertension. Med. Clin. North Am. 88:115-130.

10. Boluyt, M.O., et al. 1994. Alterations in cardiac gene expression during the transition from stable hypertrophy to heart failure. Marked upregulation of genes encoding extracellular matrix components. Circ. Res. 75:23-32.

11. Caulfield, J.B., and Borg, T.K. 1979. The collagen network of the heart. Lab. Invest. 40:364-372.

12. Rossi, M.A. 1998. Pathologic fibrosis and connective tissue matrix in left ventricular hypertrophy due to chronic arterial hypertension in humans J. Hypertens. 16:1031-1041.

13. Janicki, J.S., and Brower, G.L. 2002. The role of myocardial fibrillar collagen in ventricular remodeling and function. J. Card. Fail. 8(Suppl. 6):S319-S325.

14. Iwanaga, Y., et al. 2002. Excessive activation of matrix metalloproteinases coincides with left ventricular remodeling during transition from hypertrophy to heart failure in hypertensive rats. J. Am Coll. Cardiol. 39:1384-1391.
15. Weber, K.T., Pick, R., Jalil, J.E., Janicki, J.S., and Carroll, E.P. 1989. Patterns of myocardial fibrosis. J. Mol. Cell. Cardiol. 21(Suppl. 5):121-131.

16. Weber, K.T. 1989. Cardiac interstitium in health and disease: the fibrillar collagen network. J. Am. Coll. Cardiol. 13:1637-1652.

17. Weber, K.T., Swamynathan, S.K., Guntaka, R.V., and Sun, Y. 1999. Angiotensin II and extracellular matrix homeostasis. Int. J. Biochem. Cell Biol. 31:395-403.

18. Swaney, J.S., et al. 2005. Inhibition of cardiac myofibroblast formation and collagen synthesis by activation and overexpression of adenylyl cyclase. Proc. Natl. Acad. Sci. U. S. A. 102:437-442.

19. Rosenkranz, S. 2004. TGF-beta1 and angiotensin networking in cardiac remodeling. Cardiovasc. Res. 63:423-432.

20. Olsen, M.H., et al. 2005. Markers of collagen synthesis is related to blood pressure and vascular hypertrophy: a LIFE substudy. J. Hum. Hypertens. 19:301-307.

21. Wei, S., Chow, L.T., Shum, I.O., Qin, L., and Sanderson, J.E. 1999. Left and right ventricular collagen type I/III ratios and remodeling post-myocardial infarction. J. Card. Fail. 5:117-126.

22. Diez, J., and Hernandez, M. 1996. Is the extracellular degradation of collagen type I fibers depressed in spontaneously hypertensive rats with myocardial fibrosis? Circulation. 94:2998.

23. Diez, J., et al. 1996. Serum markers of collagen type I metabolism in spontaneously hypertensive rats: relation to myocardial fibrosis. Circulation. 93:1026-1032.

24. Norton, G.R., et al. 1997. Myocardial stiffness is attributed to alterations in cross-linked collagen rather than total collagen or phenotypes in spontaneously hypertensive rats. Circulation. 96:1991-1998.

25. Gordon, E.E., Kira, Y., Demers, L.M., and Morgan, H.E. 1986. Aortic pressure as a determinant of cardiac protein degradation. Am. J. Physiol. 250:C932-C938.

26. Morgan, H.E., Siehl, D., Chua, B.H., and Lautensack-Belser, N. 1985. Faster protein and ribosome synthesis in hypertrophying heart. Basic Res. Cardiol. 80(Suppl. 2):115-118.

27. Moalic, J.M., Bercovici, J., and Swynghedauw, B. 1984. Myosin heavy chain and actin fractional rates of synthesis in normal and overload rat heart ventricles. J. Mol. Cell. Cardiol. 16:875-884.

28. Lopez, B., Gonzalez, A., Querejeta, R., Larman, M., and Diez, J. 2006. Alterations in the pattern of col- lagen deposition may contribute to the deterioration of systolic function in hypertensive patients with heart failure. J. Am. Coll. Cardiol. 48:89-96.

29. Ahmed, S.H., et al. 2006. Matrix metalloproteinases/tissue inhibitors of metalloproteinases: relationship between changes in proteolytic determinants of matrix composition and structural, functional, and clinical manifestations of hypertensive heart disease. Circulation. 113:2089-2096.

30. Nagase, H., Visse, R., and Murphy, G. 2006. Structure and function of matrix metalloproteinases and TIMPs. Cardiovasc. Res. 69:562-573.

31. Inokubo, Y., et al. 2001. Plasma levels of matrix metalloproteinase- 9 and tissue inhibitor of metalloproteinase- 1 are increased in the coronary circulation in patients with acute coronary syndrome. Am. Heart J. 141:211-217.

32. Feldman, A.M., Li, Y.Y., and McTiernan, C.F. 2001. Matrix metalloproteinases in pathophysiology and treatment of heart failure. Lancet. 357:654-655.

33. Galis, Z.S., and Khatri, J.J. 2002. Matrix metalloproteinases in vascular remodeling and atherogenesis: the good, the bad, and the ugly. Circ. Res. 90:251-262.

34. Rucklidge, G.J., Milne, G., McGaw, B.A., Milne, E., and Robins, S.P. 1992. Turnover rates of different collagen types measured by isotope ratio mass spectrometry. Biochim. Biophys. Acta. 1156:57-61.

35. Kassiri, Z., et al. 2005. Combination of tumor necrosis factor-alpha ablation and matrix metalloproteinase inhibition prevents heart failure after pressure overload in tissue inhibitor of metalloproteinase-3 knock-out mice. Circ. Res. 97:380-390.

36. Kassiri, Z., and Khokha, R. 2005. Myocardial extracellular matrix and its regulation by metalloproteinases and their inhibitors. Thromb. Haemost. 93:212-219.

37. Mujumdar, V.S., Smiley, L.M., and Tyagi, S.C. 2001. Activation of matrix metalloproteinase dilates and decreases cardiac tensile strength. Int. J. Cardiol. 79:277-286.

38. Wang, T.L., Yang, Y.H., Chang, H., and Hung, C.R. 2004. Angiotensin II signals mechanical stretchinduced cardiac matrix metalloproteinase expression via JAK-STAT pathway. J. Mol. Cell. Cardiol. 37:785-794.

39. Matsusaka, H., et al. 2006. Targeted deletion of matrix metalloproteinase 2 ameliorates myocardial remodeling in mice with chronic pressure overload. 
Hypertension. 47:711-717.

40. Heymans, S., et al. 2005. Loss or inhibition of uPA or MMP-9 attenuates LV remodeling and dysfunction after acute pressure overload in mice. Am. J. Pathol. 166:15-25.

41. Shen, W.L., et al. 2006. NAD(P)H oxidase-derived reactive oxygen species regulate angiotensin-II induced adventitial fibroblast phenotypic differentiation. Biochem. Biophys. Res. Commun. 339:337-343.

42. Graham, H.K., and Trafford, A.W. 2006. Spatial disruption and enhanced degradation of collagen with the transition from compensated ventricular hypertrophy to symptomatic congestive heart failure. Am. J. Physiol. Heart Circ. Physiol. doi:10.1152/ajpheart.00355.2006.

43. Peterson, J.T., et al. 2001. Matrix metalloproteinase inhibition attenuates left ventricular remodeling and dysfunction in a rat model of progressive heart failure. Circulation. 103:2303-2309.

44. Sakata, Y., et al. 2004. Activation of matrix metalloproteinases precedes left ventricular remodeling in hypertensive heart failure rats: its inhibition as a primary effect of Angiotensin-converting enzyme inhibitor. Circulation. 109:2143-2149.

45. Laviades, C., et al. 1998. Abnormalities of the extracellular degradation of collagen type I in essential hypertension. Circulation. 98:535-540.

46. Lindsey, M.L., et al. 2006. Matrix metalloproteinase-7 affects connexin-43 levels, electrical conduction, and survival after myocardial infarction. Circulation. 113:2919-2928.

47. Li, Y.Y., McTiernan, C.F., and Feldman, A.M. 2000. Interplay of matrix metalloproteinases, tissue inhibitors of metalloproteinases and their regulators in cardiac matrix remodeling. Cardiovasc. Res. 46:214-224.

48. Lopez, B., Gonzalez, A., and Diez, J. 2004. Role of matrix metalloproteinases in hypertension-associated cardiac fibrosis. Curr. Opin. Nephrol. Hypertens. 13:197-204.

49. Senior, R.M., Griffin, G.L., and Mecham, R.P. 1980 Chemotactic activity of elastin-derived peptides. J. Clin. Invest. 66:859-862.

50. Faury, G., et al. 1998. Action of tropoelastin and synthetic elastin sequences on vascular tone and on free $\mathrm{Ca} 2+$ level in human vascular endothelial cells. Circ. Res. 82:328-336.

51. Manso, A.M., Elsherif, L., Kang, S.M., and Ross, R.S. 2006. Integrins, membrane-type matrix metalloproteinases and ADAMs: potential implications for cardiac remodeling. Cardiovasc. Res. 69:574-584.

52. Katsumi, A., Naoe, T., Matsushita, T., Kaibuchi, K., and Schwartz, M.A. 2005. Integrin activation and matrix binding mediate cellular responses to mechanical stretch. J. Biol. Chem. 280:16546-16549.

53. Ginsberg, M.H., Partridge, A., and Shattil, S.J. 2005. Integrin regulation. Curr. Opin. Cell Biol. 17:509-516.

54. De Acetis, M., et al. 2005. Cardiac overexpression of melusin protects from dilated cardiomyopathy due to long-standing pressure overload. Circ. Res.
96:1087-1094.

55. Risinger, G.M., Jr., Hunt, T.S., Updike, D.L., Bullen, E.C., and Howard, E.W. 2006. Matrix metalloproteinase-2 expression by vascular smooth muscle cells is mediated by both stimulatory and inhibitory signals in response to growth factors. J. Biol. Chem. 281:25915-25925.

56. Maeda, M., et al. 2003. Dystrophin upregulation in pressure-overloaded cardiac hypertrophy in rats. Cell Motil. Cytoskeleton. 55:26-35.

57. Kamogawa, Y., et al. 2001. Dystrophin-deficient myocardium is vulnerable to pressure overload in vivo. Cardiovasc. Res. 50:509-515.

58. Cohn, R.D. 2005. Dystroglycan: important player in skeletal muscle and beyond. Neuromuscul. Disord. 15:207-217.

59. Miner, J.H., et al. 1997. The laminin alpha chains: expression, developmental transitions, and chromosomal locations of alpha1-5, identification of heterotrimeric laminins 8-11, and cloning of a novel alpha3 isoform. J. Cell Biol. 137:685-701.

60. Oak, S.A., Zhou, Y.W., and Jarrett, H.W. 2003. Skeletal muscle signaling pathway through the dystrophin glycoprotein complex and Rac1. J. Biol. Chem. 278:39287-39295.

61. Tomasek, J.J., Gabbiani, G., Hinz, B., Chaponnier, C., and Brown, R.A. 2002. Myofibroblasts and mechano-regulation of connective tissue remodelling. Nat. Rev. Mol. Cell Biol. 3:349-363.

62. Schiller, M., Verrecchia, F., and Mauviel, A. 2003. Cyclic adenosine $3^{\prime}, 5^{\prime}$-monophosphate-elevating agents inhibit transforming growth factor-betainduced SMAD3/4-dependent transcription via a protein kinase A-dependent mechanism. Oncogene. 22:8881-8890

63. Wang, W., et al. 2006. Essential role of Smad3 in angiotensin II-induced vascular fibrosis. Circ. Res. 98:1032-1039

64. Sorescu, D. 2006. Smad3 mediates angiotensin IIand TGF-beta1-induced vascular fibrosis: $\mathrm{Smad} 3$ thickens the plot. Circ. Res. 98:988-989.

65. Huang, Y., et al. 2006. Renin increases mesangial cell transforming growth factor-beta 1 and matrix proteins through receptor-mediated, angiotensin IIindependent mechanisms. Kidney Int. 69:105-113.

66. Mehta, P.K., and Griendling, K.K. 2007. Angiotensin II cell signaling: physiological and pathological effects in the cardiovascular system. Am. J. Physiol. Cell Physiol. 292:C82-C97.

67. Bagrodia, S., D’Erijard, B., Davis, R.J., and Cerione, R.A. 1995. Cdc42 and PAK-mediated signaling leads to Jun kinase and p38 mitogen-activated protein kinase activation. J. Biol. Chem. 270:27995-27998.

68. Schmitz, U., Thommes, K., Beier, I., Dusing, R., and Vetter, H. 2004. Identification of Nck interacting proteins in vascular smooth muscle cells. Clin. Exp. Hypertens. 26:267-275.

69. Eguchi, S., et al. 2001. Activation of MAPKs by angiotensin II in vascular smooth muscle cells.
Metalloprotease-dependent EGF receptor activation is required for activation of ERK and 338 MAPK but not for JNK. J. Biol. Chem. 276:7957-7962.

70. Schafer, B., Marg, B., Gschwind, A., and Ullrich, A. 2004. Distinct ADAM metalloproteinases regulate $\mathrm{G}$ protein coupled receptor-induced cell proliferation and survival. J. Biol. Chem. 279:47929-47938.

71. Yoshioka, J., et al. 2005. Cardiomyocyte hypertrophy and degradation of connexin 43 through spatially restricted autocrine/paracrine heparin-binding EGF. Proc. Natl. Acad. Sci. U. S. A. 102:10622-10627.

72. Hyytiainen, M., Penttinen, C., and Keski-Oja, J. 2004. Latent TGF-beta binding proteins: extracellular matrix association and roles in TGFbeta activation. Crit. Rev. Clin. Lab. Sci. 41:233-264.

73. Ge, G., and Greenspan, D.S. 2006. BMP1 controls TGFbeta1 activation via cleavage of latent TGFbeta-binding protein. J. Cell Biol. 175:111-120.

74. Vasan, R.S., et al. 2004. Relations of serum aldosterone to cardiac structure: gender-related differences in the Framingham Heart Study. Hypertension. 43:957-962.

75. Jaffe, I.Z., and Mendelsohn, M.E. 2005. Angiotensin II and aldosterone regulate gene transcription via functional mineralocortocoid receptors in human coronary artery smooth muscle cells. Circ. Res. 96:643-650.

76. Fiebeler, A., et al. 2001. Mineralocorticoid receptor affects AP-1 and nuclear factor-kappab activation in angiotensin II-induced cardiac injury. Hypertension. 37:787-793.

77. Susic, D., Varagic, J., Ahn, J., Matavelli, L.C., and Frohlich, E.D. 2006. Long-term mineralocorticoid receptor blockade reduces fibrosis and improves cardiac performance and coronary hemodynamics in elderly SHR. Am. J. Physiol. Heart Circ. Physiol. 292: $\mathrm{H} 175-\mathrm{H} 179$.

78. Li, R.K., et al. 1997. Overexpression of transforming growth factor-beta 1 and insulin-like growth factor-I in patients with idiopathic hypertrophic cardiomyopathy. Circulation. 96:874-881.

79. Eghbali, M., Tomek, R., Woods, C., and Bhambi, B. 1991. Cardiac fibroblasts are predisposed to convert into myocyte phenotype: specific effect of transforming growth factor beta. Proc. Natl. Acad. Sci. U. S. A. 88:795-799.

80. Desmouliere, A., Geinoz, A., Gabbiani, F., and Gabbiani, G. 1993. Transforming growth factor-beta 1 induces alpha-smooth muscle actin expression in granulation tissue myofibroblasts and in quiescent and growing cultured fibroblasts. J. Cell Biol. 122:103-111.

81. Rosenkranz, S., et al. 2002. Alterations of betaadrenergic signaling and cardiac hypertrophy in transgenic mice overexpressing TGF-beta(1). Am.J. Physiol. Heart Circ. Physiol. 283:H1253-H1262.

82. Spence, H.J., Dhillon, A.S., James, M., and Winder, S.J. 2004. Dystroglycan, a scaffold for the ERKMAP kinase cascade. EMBO Rep. 5:484-489. 